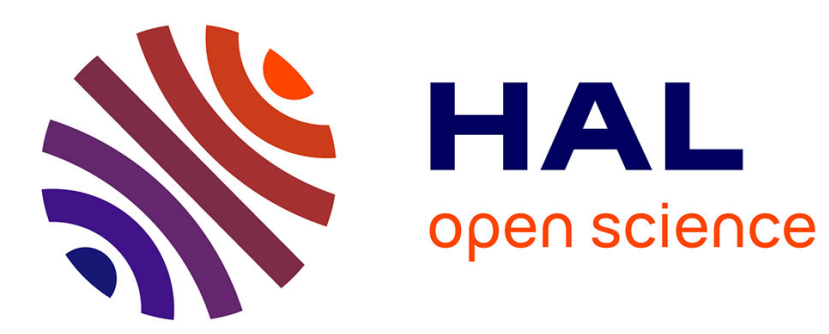

\title{
On the Construction of Load-Balanced (k,r-hop)-Connected Dominating Set for WSNs
}

Tarek Moulahi, Hervé Guyennet, Salem Nasri, Rjab Hajlaoui

\section{To cite this version:}

Tarek Moulahi, Hervé Guyennet, Salem Nasri, Rjab Hajlaoui. On the Construction of Load-Balanced (k,r-hop)-Connected Dominating Set for WSNs. ANTS 2012, IEEE Int. Conf. on Advanced Networks and Telecommunications Systems, Jan 2012, India. pp.76 - 80. hal-00940604

\section{HAL Id: hal-00940604 https://hal.science/hal-00940604}

Submitted on 2 Feb 2014

HAL is a multi-disciplinary open access archive for the deposit and dissemination of scientific research documents, whether they are published or not. The documents may come from teaching and research institutions in France or abroad, or from public or private research centers.
L'archive ouverte pluridisciplinaire HAL, est destinée au dépôt et à la diffusion de documents scientifiques de niveau recherche, publiés ou non, émanant des établissements d'enseignement et de recherche français ou étrangers, des laboratoires publics ou privés. 


\section{On the Construction of Load-Balanced (k,r-hop)- Connected Dominating Set for WSNs}

\author{
Tarek Moulahi ${ }^{1,2}$, Hervé Guyennet ${ }^{1}$ \\ ${ }^{1}$ University of Besançon \\ Besançon, France \\ tarek.moulahi@lifc.univ-fcomte.fr \\ herve.guyennet@lifc.univ-fcomte.fr
}

\author{
Salem Nasri ${ }^{2}$, Rjab Hajlaoui ${ }^{2}$ \\ ${ }^{2}$ ENIS, University of Sfax \\ Sfax, Tunisia \\ salem.nasri@enim.rnu.tn \\ hajlaoui.rjab@gmail.com
}

\begin{abstract}
Connected Dominating Sets (CDS) are selected to construct a virtual backbone in Wireless Sensor Networks (WSNs). Indeed, CDS are used for fault tolerant broadcasting, routing and even efficient gathering of data. On the other hand, r-hop connected k-dominating sets ((k,r-hop)-CDS) are introduced to reduce the size of CDS and the total amount of information to be transmitted. Unfortunately, no work considers the load-balance factor of $(\mathrm{k}, \mathrm{r}$-hop)-CDS. Recently, there is a proposition of constructing load-balanced CDS for WSNs. In this paper we re-use the same concept of selecting load-balanced CDS to construct a Load-Balanced (k,r-hop)-CDS (LB(k,r-hop)-CDS). Then, the allocation of dominatees to the appropriate dominators is done. Through simulations, the proposed method of constructing $L B(k, r-h o p)-C D S$ extends network lifetime by an important rate compared with the most recent (k,r-hop)-CDS construction method.
\end{abstract}

Keywords-component; Load-balancing, (k,r-hop)-CDS, Virtual backbone, Fault tolerance,WSNs.

\section{INTRODUCTION}

WSNs are ad hoc networks composed of many sensor nodes supervised and controlled by a base station. Each sensor is a small device able to sense, to compute and to communicate via wireless channel. The low cost of these devices and the functionalities of collecting, computing and communicating data have attracted the attention of the community. Thus, WSNs are actually used in several domains such us military, health care, environment and others [1,2]. Nodes in WSNs are deployed without any predefined topologies. Consequently, this network is formed randomly using wireless radio channels. The most important disadvantage of WSNs is their limited energetic capacity, so that many methods, algorithms and protocols were introduced and developed taking in consideration this constraint.

Moreover, the main functionality of WSNs is to carry out a collaborative work in order to observe and supervise nature. That is why, the communication between nodes has a major importance. According to the energy constraint in sensors, many heuristics and strategies were proposed to minimize the broadcasting storm problem, the redundancy $[3,4]$ and to realize a communication with a respectable level of fault tolerance. Selecting a CDS provide a virtual backbone used when broadcasting, routing or even for gathering data. A (k,r-
hop)-CDS is introduced to reduce the size of CDS and the amount of information to be transmitted. In fact, a (k,r-hop)CDS problem is to select a connected set $D$ of the network respecting that every vertex $u$ not in $D$ is at a distance within rhop from at list $k$ vertices in $D$. Recently, there is a proposition to find out a load-balanced CDS $[12,13,14]$ that helps to extend the network lifetime with a very high rate. According to the best of our knowledge there is no a proposition of LB(k,r-hop)CDS. The goal behind our work is to provide a solution to resolve this problem.

This paper is organized as follows. In the next section, we give some important definitions to clarify the vocabularies used in this work. In section III, there is a study of the related work. Section IV describes the problem definition. In section V, the main problem is treated. Section VI contains some measurement and experimental results to illustrate our improvement. Finally, section VII is a recapitulation of this work.

\section{DEFINITIONS}

In this section, we present some important definitions that will be used later.

\section{A. Network model}

In this paper we only consider unit disk graph, static WSNs and sensors with the same coverage area. WSNs can be considered as a graph $G(V ; E)$, where $V$ is the set of vertex representing nodes and $E$ is the set of edges between vertexes. An edge exists between two nodes $A$ and $B$ in this graph only if $A$ is in the coverage area of $B$ and $B$ is in the coverage area of $A$.

\section{B. $\quad k$-connected graph}

A graph $G(V ; E)$ is said k-connected graph if and only if it contains $\mathrm{k}$ independent paths between any two vertices.

\section{C. $\quad k$-connected m-dominating sets}

A subset $C$ of $V$ is k-connected m-dominating set if every node in $V \backslash C$ is dominated by at least m nodes in $C$ and the sub graph induced by $\mathrm{C}$ is k-connected, The nodes in $C$ are denoted as dominators, and nodes in $V \backslash C$ are denoted as dominatees. 
$D$.

r-hop connection

Given two vertexes $\mathrm{u}$ and $\mathrm{v}$ of a graph $G(V ; E), \mathrm{u}$ is r-hop connected by $\mathrm{v}$ if we can find a path in $G$ from $\mathrm{u}$ to $\mathrm{v}$ within $\mathrm{r}$ hops i.e. this path has $\mathrm{r}-1$ intermediates nodes.

E.

$r$-hop connected $k$-dominating sets

A subset $D$ of $V$ is r-hop connected kdominating set if every node in $V \mid D$ is $r$-hop dominated by at least $\mathrm{k}$ nodes in $D$ and the sub graph induced by $D$ is connected, The nodes in $D$ are denoted as dominators, and node in $V \mid D$ are denoted as dominatees.

\section{RELATED WORK}

To construct a CDS, lots of efforts have been made and many approaches were used either centralized or decentralized. In what follows, a description of the most important methods of CDS selection are given in addition a short study of the recent LBCDS problem is realized.

\section{A. CDS-based backbone}

Many heuristic were proposed to resolve the problem of CDS construction. In [5], the authors provide a method of CDS selection based on timer that can be applied to Mobile Ad Hoc Networks (MANET). The construction of CDS can be either centralized or decentralized which mean either that one hope select the CDS or that the whole network participates to the CDS selection. In [6] there is a proposition of a decentralized CDS construction method for MANET. In [8] a method of searching minimum $\mathrm{m}$-connected $\mathrm{k}$-tuple $\mathrm{CDS}$ is given, this method helps to reduce the consumed energy in routing. The $(\mathrm{k}, \mathrm{m}) \mathrm{CDS}$ helps to realize the task of routing or broadcasting with high level of fault tolerance [7]. In fact, every node out of the CDS is dominated by $\mathrm{m}$ node in CDS and nodes in CDS are k-connected.

$B$.

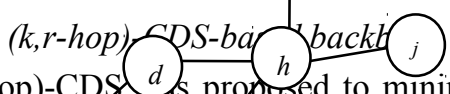

(k,r-hop)-CDs $d$ s proposed to minimize the information to be transmitted as wdll as the cost of communication. In [10]

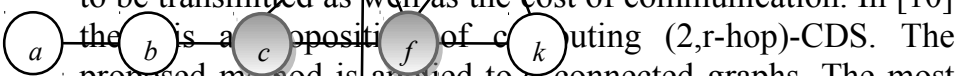

proposed mongod is appried to z-connected graphs. The most recent heuristic $\mathrm{k}, \mathrm{r}-\mathrm{h} y$ CDS was proposed in [11]. Indeed, this heurile is de $i$ kaliza and helps to extract a virtual backbone in two steps: firs $l$. cluster construction is done by selecting (k,r-hop)DS from the graph. Next, (k,rhop)DS are linked to each other to form (k,r-hop)-CDS.

\section{Load-balanced CDS-based backbone}

Recently, Jing $\mathrm{He}$ et al. proposed some methods to construct a load-balanced CDS. In [12], they make use of a genetic algorithm to select LBCDS. In [13] they propose a LBCDS based on a heuristic which is done in two steps. First, the selection of dominators is done. Next, they define a loadbalanced method for allocation of the dominatees to the appropriate dominators. This operation allows the increasing of network lifetime. In [14], they used an evolutionary algorithm to resolve the LBCDS problem.
D. Synthesis

To the best of our knowledge there is no works that consider the load-balanced factor when constructing (k,r-hop)CDS. In this paper we try to re-use the concepts recently defined in $[12,13,14]$ to resolve the problem of $\mathrm{LB}(\mathrm{k}, \mathrm{r}-\mathrm{hop})$ CDS.

\section{Problem Definition}

In this section, we try to give the definition of our problem by studying a concrete example.

\section{A. Symbols and notations}

In table I, we introduce the symbols used in this paper and their definitions.

TABLE I. SYMBOLS AND NOTATIONS

\begin{tabular}{|l|l|}
\hline Symbol & Signification \\
\hline$a, b, c \ldots .$. & Refers to the sensors used in the network \\
\hline$N D(x)$ & Refers to the set of dominatees of a dominator $x$ \\
\hline$|N D(x)|$ & The number of element in $N D(x)$ \\
\hline$p$-norm & $\begin{array}{l}\text { Defined to compute the load-balanced factor of a } \\
\text { subset in WSNs, proposed in [15], and used in } \\
{[12,13] \text { it is equal to: }}\end{array}$ \\
\hline$d(x)$ & $|\mathbf{X}|_{p}=\left(\sum_{n=1}^{n}\left|x_{i}\right|^{p}\right)^{1 / p}$ \\
\hline $\bar{d}$ & $\begin{array}{l}\text { Refers to the degree of a node } x \text { in the graph } \\
\text { of degrees in a graph } G .\end{array}$ \\
\hline$D$ & The set of dominators in a graph $G$ \\
\hline
\end{tabular}

\section{B. Dominators selection}

In fig. 1, an example of WSNs is given. The (1,2-hop)-CDS from the given network is selected using the latest algorithm of (k,r-hop)-CDS construction proposed in [11]. The result of this selection is the set $\{c, f\}$.

Figure 1. Example of a WSN : (1,2-hop)CDS is $\{\mathrm{c}, \mathrm{f}\}$

In order to measure the load-balancing of the selected set we compute its $p$-norm. According to [15], for $\mathrm{p}=2$ the data flow shows an analogy to electrostatic filed. So, computing $p$ norm with $\mathrm{p}=2$ helps to measure the load-balance among $x_{i}$. Finally, if the p-norm of a set becomes small this set will be more load-balanced [12]. According to $[12,13]$ :

$$
x_{i}=\left|d_{i}-\bar{d}\right|
$$

Hence,

$p-\operatorname{norm}(\{c, f\})=\sqrt{|4-3|^{2}+|6-3|^{2}}=\sqrt{10}$ 
In fig. 2, we try to select another (1,2-hop)-CDS which is $\{c, d, e\}$. For this set the $p$-norm will be:

$p-\operatorname{norm}(\{c, d, e\})=\sqrt{|4-3|^{2}+|4-3|^{2}+|4-3|^{2}}=\sqrt{3}$

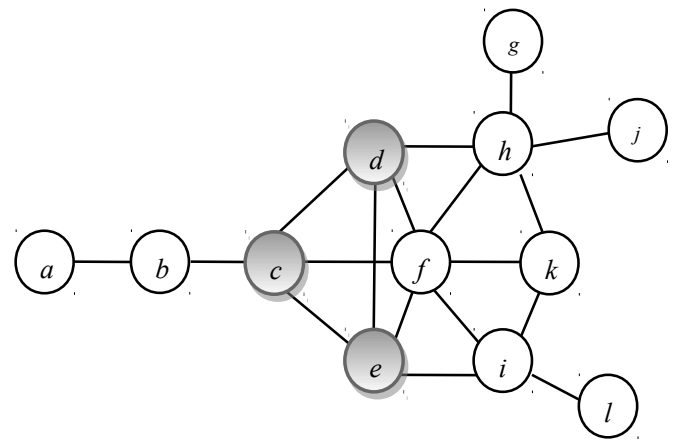

Figure 2. Example of a WSN: (1,2-hop)CDS is $\{\mathrm{c}, \mathrm{d}, \mathrm{e}\}$

After a simple comparison of the two p-norms:

$$
\sqrt{10} \hbar \sqrt{3}
$$

To conclude, we can say that the second set is more loadbalanced. Consequently, constructing a LB(k,r-hop)-CDS should take into consideration the minimization of its p-norm value.

\section{Dominatees association}

In this subsection, we give two examples of dominatees' association to dominators. Next, we compare the two possibilities to see which of them is more load-balanced. In fig. $3, N D(c)=\{b, a\} ; N D(d)=\{h, g, j, f, k\}$ and $N D(e)=\{i, l\}$.

:Then, if we choose

$$
x_{i}=|N D(i)|-\overline{N D(i)} \mid
$$

Hence,

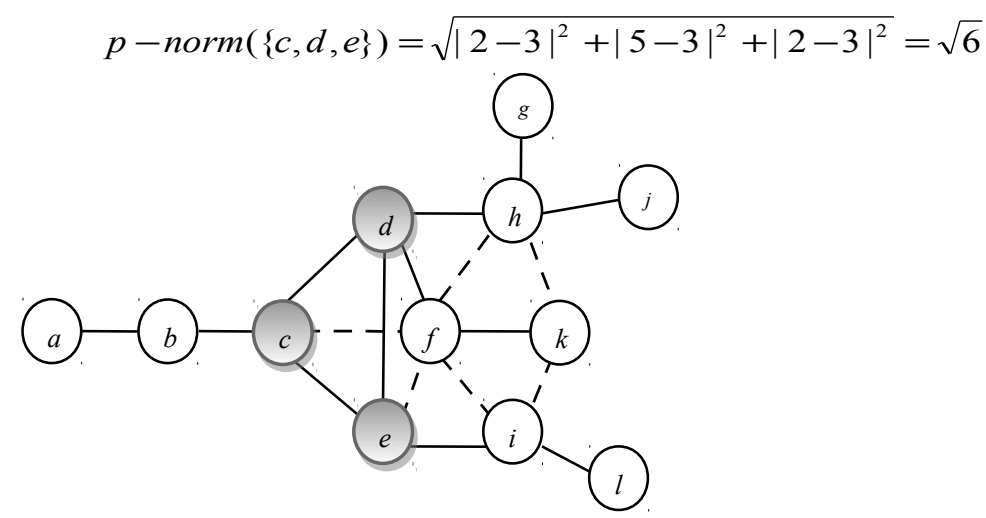

Figure 3. Example of a WSN: a non equilibrate association of dominatees to dominators

In fig. 4, another association of dominatees to dominators is done: $N D(c)=\{b, a\} ; N D(d)=\{h, g, j\}$ and $N D(e)=\{i, l, f, k\}$. Then the $p$-norm will be:

$p-\operatorname{norm}(\{c, d, e\})=\sqrt{|2-3|^{2}+|3-3|^{2}+|4-3|^{2}}=\sqrt{2}$

Finally, we can deduce $\mathrm{t} g$ he second association is more load-balanced.

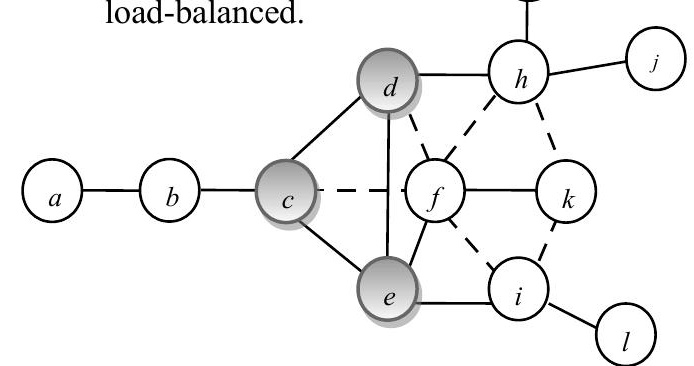

Figure 4. Example of a WSN: an equilibrate association of dominatees to dominators

\section{V. $\quad \mathrm{LB}(\mathrm{K}, \mathrm{R}-\mathrm{HOP})-\mathrm{CDS}$}

In this section, the main problem is formally treated. Two steps are done to resolve LB(k,r-hop)-CDS problem. First, the dominators are selected. Next, the association of dominatees to dominators is done. We treat only $\mathrm{LB}(1, \mathrm{r}-\mathrm{hop})$-CDS.

\section{A. CDS selection}

According to the example studied in the previous section the construction of CDS is to select a subset $D$ of $V(V$ is the set of vertices of the WSNs represented by the graph $G(V, E)$ ). With $D$ should has the next mentioned proprieties

- $\quad D$ is connected.

- For all node $u$ of $V \mid D$, there is a node $v$ in $D$, such that $u$ is at a distance within r-hop from $v$.

- Minimize $|D|_{p}=\left(\sum_{=1}^{D \mid}\left|d\left(x_{i}\right)-\bar{d}\right|^{2}\right)^{1 / 2}$

Thus, the selection of $D$ should start by selecting node that minimizes $|D|_{p}$ and it ended when $D$ is connected, and every node not in $D$ is at a distance within r-hop from at least one node in $D$. Finally, we optimize $D$ i.e. extracting node $n$ from $D$, if $D \mid\{n\}$ still respect the above proprieties. Below, the algorithm of $D$ selection is given:

\begin{tabular}{l}
\hline \multicolumn{1}{c}{ Algorithm1: Dominators selection } \\
\hline Input: a connected graph $G(V, E)$ \\
Output: the set of dominators $D$ \\
1: $D \leftarrow \varnothing$ \\
2: compute $\bar{d}$ in $G$ \\
3: for all node $n$ in $G$ do \\
4: $\quad$ if $d(n)=\bar{d}$ then $D \leftarrow D U\{n\}$ \\
5: if $D$ is connected and all nodes in $V \mid D$ are within $\mathrm{r}$ - \\
6: hop from at least one node in $D$ then \\
7: $\quad$ else $\bar{d} \leftarrow \bar{d}+1$ \\
8: $\quad$ end if \\
9: end for all \\
10: for all node $n$ in $D$ do \\
11: if $D \backslash\{n\}$ is connected and all nodes in $V \mid D \backslash\{n\}$ are \\
$\quad$ within $\mathrm{r}$-hop from at least one node in $D \mid\{n\}$ then \\
12: $\quad D \leftarrow D \backslash\{n\}$ \\
13: end if \\
14: end for all
\end{tabular}


If we apply algorithm 1 to the network of fig. 1 in order to construct (1,2-hop)CDS, we have $\bar{d}=3$, there is no node having 3 neighbors. Then, for $\bar{d}=4, D=\{c\}$. For this set $D$ there still exist uncovered nodes within 2-hops. Next, $D=\{c, d\}$ this set also does not cover all nodes within 2-hops. Finally, $D=\{c, d, e\}$. This $D$ is connected and all nodes in $V \backslash D$ are within 2-hops from at least one node in $D$. Consequently, the algorithm terminates. If $i$ is chosen as an element of $D$, it will be extracted in the step of optimization before the end of the algorithm.

\section{B. Load-balanced allocation of dominatees to dominators}

The problem of load-balanced allocation of dominatees to dominators can be defined as:

- Every dominatees is allocated to only one domiantors.

- The distance between a dominatee and his allocated dominator is within $r$ hops.

- The allocation should minimize

$$
|D|_{p}=\left(\sum_{=1}^{D \mid} \| N D\left(x_{i}\right)\left|-\overline{N D\left(x_{i}\right)}\right|^{2}\right)^{1 / 2}
$$

The algorithm below is defined respecting the above mentioned properties. The algorithm is started by the allocation of the imposed dominatees i.e.:

- A node that can be allocated only to one dominator.

- Or it is a neighbor of only one dominator.

- Or it is a neighbor of only one allocated dominatee.

For other nodes the association is done to the dominators having the minimum $|N D(x)|$

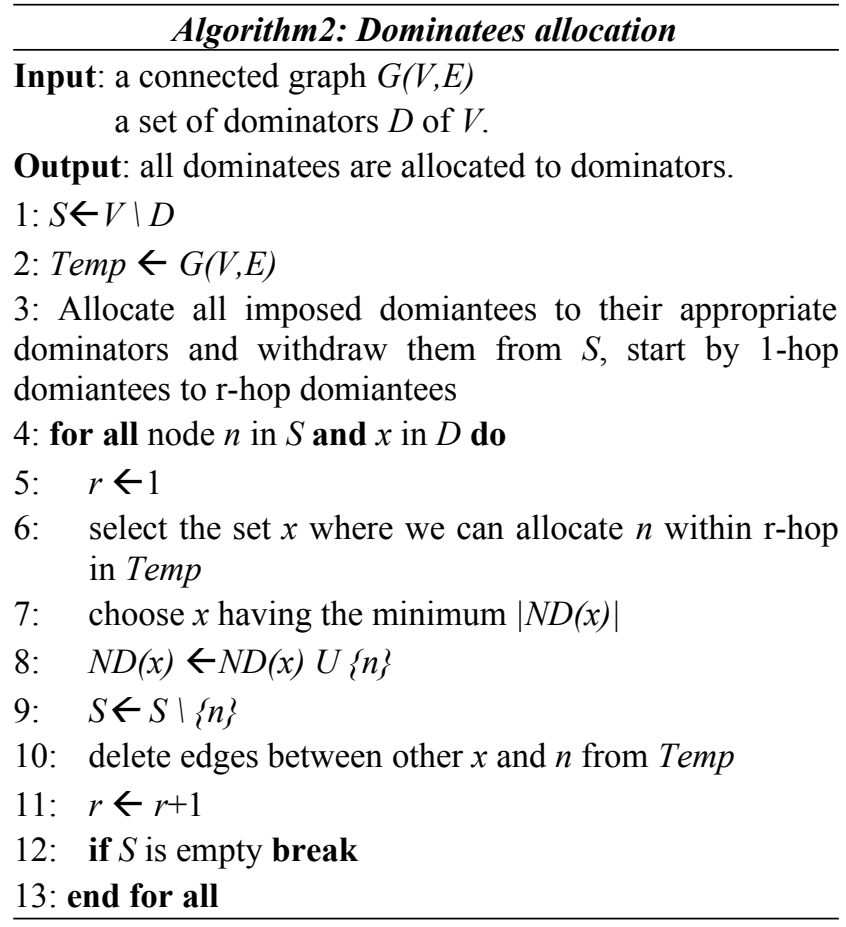

If we apply algorithm 2 to the network of fig. 2 in order to complete the allocation of dominatees to dominators. First,
$N D(c)=\{a, b\}$ because $a$ and $b$ are imposed to $c$. Same for $h$ and $i$ which are imposed respectively to $d$ and $e$. Then, $l$ is imposed to $e$ and $g, j$ are imposed to $d$. Consequently, $N D(d)=\{h, g, j\}$ and $N D(e)=\{i, l\}$. The node $f$ will be linked to $e$ because $|N D(e)|<|N D(f)|$. Finally, $N D(e)=\{i, l, f, k\}$ and, $\mathrm{S}$ will be empty therefore, the algorithm terminates.

\section{Performance And Evalution}

The test and the measurement of the performance are done using NS2 [16]. Identical sensors with the same coverage area $(50 \mathrm{~m})$ are used. The size of packet to be transmitted between nodes is $200 b$ or $400 b$. We suppose in this test that the sink node is directly linked to one node of the (k,r-hop)-CDS. In every 10 seconds, the sink node broadcast a packet to all nodes in the network, and after 10 seconds it receives gathered data from all nodes. Finally, we extract periodically the energy of the most worker node in the (k,r-hop)-CDS selected by our load-balanced method. Then, we compare it to the energy of the most worker node in the (k,r-hop)-CDS which is selected using the method proposed in [11]. Initially, every node is alimented by two AA lithium batteries i.e. the initial energy in nodes it is nearly $30 \mathrm{~kJ}$. We treat only the consumed energy for communication and we neglect the consumed energy for sensing and internal processing.

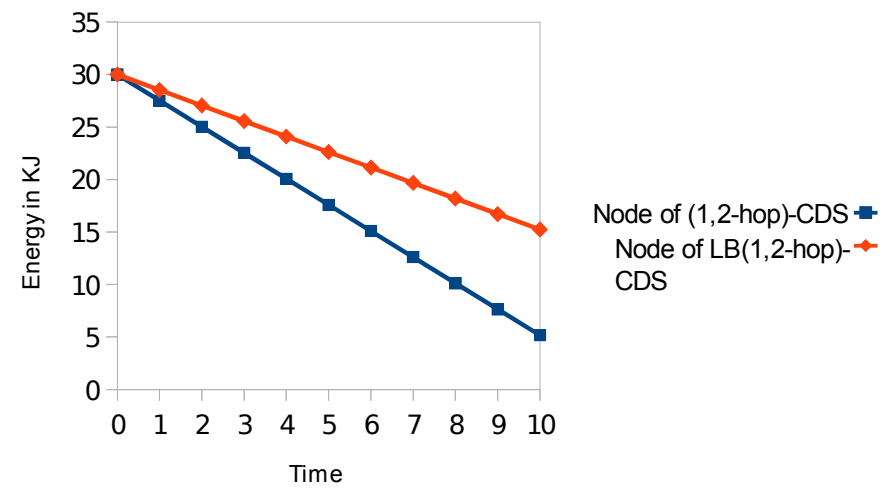

Figure 5. Evolution of energy in the most worker node in the (1,2-hop)CDS with a density of 12 nodes and using packet with $200 \mathrm{~b}$ as size

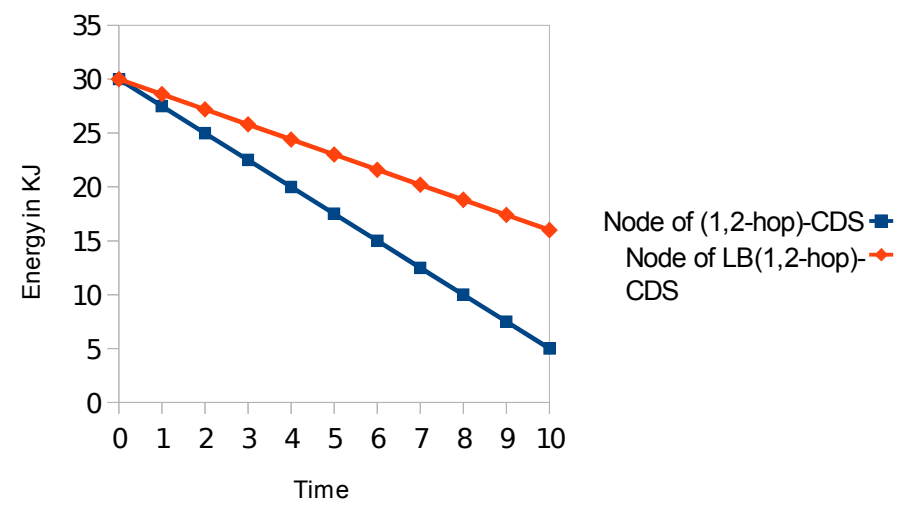


Figure 6. Evolution of energy in the most worker node in the (1,2-hop)CDS with a density of 200 nodes and using packet with $200 \mathrm{~b}$ as size

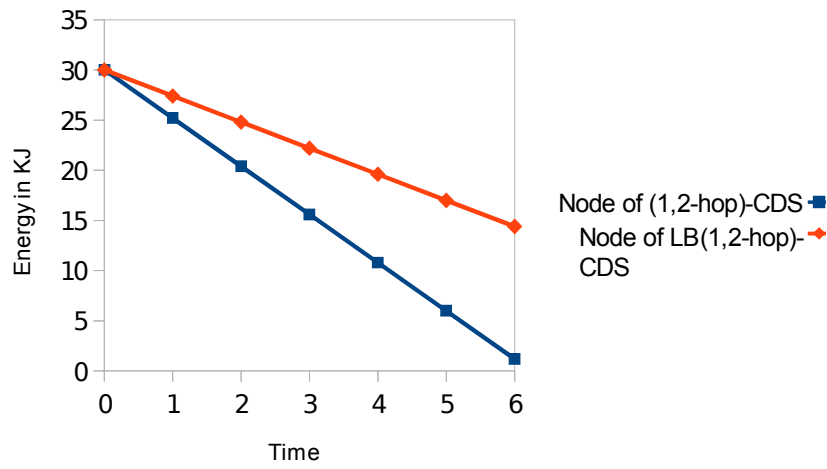

Figure 7. Evolution of energy in the most worker node in the (1,2-hop)CDS with density of 200 nodes and using packet with $400 \mathrm{~b}$ as size

In fig. 5, we can observe that there is an important improvement of the lifetime of the most worker node in the (1,2-hop)CDS. The two curves are linear. Consequently, we can easily compute the time when nodes will be exhausted. Finally, we can conclude that LB(k,r-hop)-CDS make an extension of node lifetime with $68 \%$ if we compare it with the latest proposed ( $\mathrm{k}, \mathrm{r}-\mathrm{hop})-\mathrm{CDS}$ construction method. This fact is confirmed in fig. 6 using a network with 200 nodes as density and $200 \mathrm{~b}$ as size of transmitted packet and in the network of fig. 7, with 200 nodes as density and $400 \mathrm{~b}$ as size of transmitted packet. The extension of the lifetime of the most worker node in the CDS has an important effect on extending the whole network lifetime. Indeed if a node in CDS will be quickly exhausted the CDS will be disconnected. Then a big set of sensors in the network will be unreachable.

\section{CONCLUSION}

This work treats the problem of LB(k,r-hop)-CDS aiming to select a (k,r-hop)-CDS with taking into consideration the load-balance factor. The problem resolution is centralized, is based on the minimization of p-norm and done in two steps. First, the set of dominators is selected. Next, the allocation of dominatees to the appropriate dominators is completed in order to guarantee the load-balance propriety. According to the measurement of performance given, LB(k,r-hop)-CDS provides an important improvement in term of increasing the network lifetime compared to the latest proposed method of (k,r-hop)-CDS selection.

\section{REFERENCES}

[1] Jennifer Yick, Biswanath Mukherjee and Dipak Ghosal. "Wireless sensor network survey", Computer Networks 52, pp.2292-2330, 2008 .
[2] I.F. Akyildiz, W. Su, Y. Sankarasubramaniam, E. Cayirci. "Wireless sensor networks: a survey", Computer Networks 38, pp. 393-422, 2002.

[3] Tarek Moulahi, Hervé Guyennet, Mohamed Lehsaini and Salem Nasri. "An Energy Aware MPR-based Broadcasting Algorithms for Wireless Sensor Networks", International Conference on Selected Topics in Mobile and Wireless Networking (iCOST), pp.13-18, 2011.

[4] Tarek Moulahi, Salem Nasri and Hervé Guyennet. "Amalioration of MPR by a Backbone-based Brodcasting Algorithm for WSNs", International Conference on Information Technology and eservices (ICITeS), pp.506-511, 2012.

[5] Kazuya Sakai, Scott C.-H. Huang, Wei-Shinn Ku, Min-Te Sun, and Xiuzhen Cheng, "Timer-Based CDS Construction in Wireless Ad Hoc Networks", IEEE Transactions on mobile computing, vol. 10, no. 10, pp. 1388-1402, 2011

[6] Julien Schleich, Pascal Bouvry and Le Thi Hoai An, "Decentralized Fault-tolerant Connected Dominating Set Algorithm for Mobile Ad hoc Networks", Proceedings of ICWN, pp. 354-360, 2009.

[7] Yongzhao Bian, Haibin Yu and Peng Zeng, "Construction of a fault tolerance connected dominating set in wireless sensor network", International Conference on Measuring Technology and Mechatronics Automation, pp. 610-614, 2009.

[8] Weiping Shang, Pengjun Wan, Frances Yao, Xiaodong Hu," Algorithms for minimum m-connected k-tuple dominating set problem", Theoretical Computer Science, pp.241-247, 2007.

[9] Yiwei Wu, Feng Wang, My T. Thai and Yingshu Li, "Constructing k-connected $\mathrm{m}$-dominating sets in wireless sensor networks", Military Communications Conference, IEEE, pp. 1-7, 2008.

[10] Xiuying Li and Zhao Zhang, "Two algorithms for minimum 2-connected r-hop dominating set", Information Processing Letters, Volume 110, Issue 22, 2010.

[11] Chan Zheng, Yiqing Zhang and Ling Yin, "Constructing $(\mathrm{k}, \mathrm{r})$-Connected Dominating Sets for Robust Backbone in Wireless Sensor Networks", 11th International Symposium on Communications and Information Technologies, pp. 174-177, 2011.

[12] Jing He, Shouling Ji, Mingyuan Yan, Yi Pan, and Yingshu Li, "LoadBalanced CDS Construction in Wireless Sensor Networks Via Genetic Algorithm", International Journal of Sensor Networks (IJSNET), Vol. 11, No. 3, pp(s): 166-178, 2012

[13] Jing He, Shouling Ji, Pingzhi Fan, Yi Pan, and Yingshu Li, "Constructing a Load-Balanced Virtual Backbone in Wireless Sensor Networks", ICNC 2012, Maui, Hawaii, USA, January 30-February 2, 2012.

[14] Jing He, Shouling Ji, Mingyuan Yan, Yi Pan, and Yingshu Li, "GeneticAlgorithm-Based Construction of Load-Balanced CDSs in Wireless Sensor Networks", MILCOM 2011, Baltimore, Maryland, USA, November 7-10, 2011.

[15] M. Kalantri, M. Haghpanahi, and M. Shayman. A p-norm Flow Optimaziation Problem in Dense Wireless Sensor Network, INFOCOM, 2008.

[16] The Network Simulator - ns-2, http://www.isi.edu/nsnam/ns/. 